favorable to bladder distension, is very unpleasant to the patient if she be not anesthetized, while if an anesthetic $i$ used, its administration is very awkwardly carried out. The patient's neck is apt to get much bent, and if she vomits or secretes mucus freely there is often trouble in managing these complications satisfactorily. Moreover, the position of the examiner is not apt to be at all a comfortable one, especially in catheterizing the ureters.

During the past three years the writer has employed a method which is free from these objections. The patient is placed on a Boldt operating table in the lithotomy position, the ankles being fastened to upright rods, the buttocks projecting slightly over the end of the table resting on a rubber pad. A steel bar with two padded supports is attached to the top of the table so as to support the shoulders. After the external genitals and vagina are cleansed, the patient is enveloped in sterile sheets, the urine is withdrawn from the bladder, the

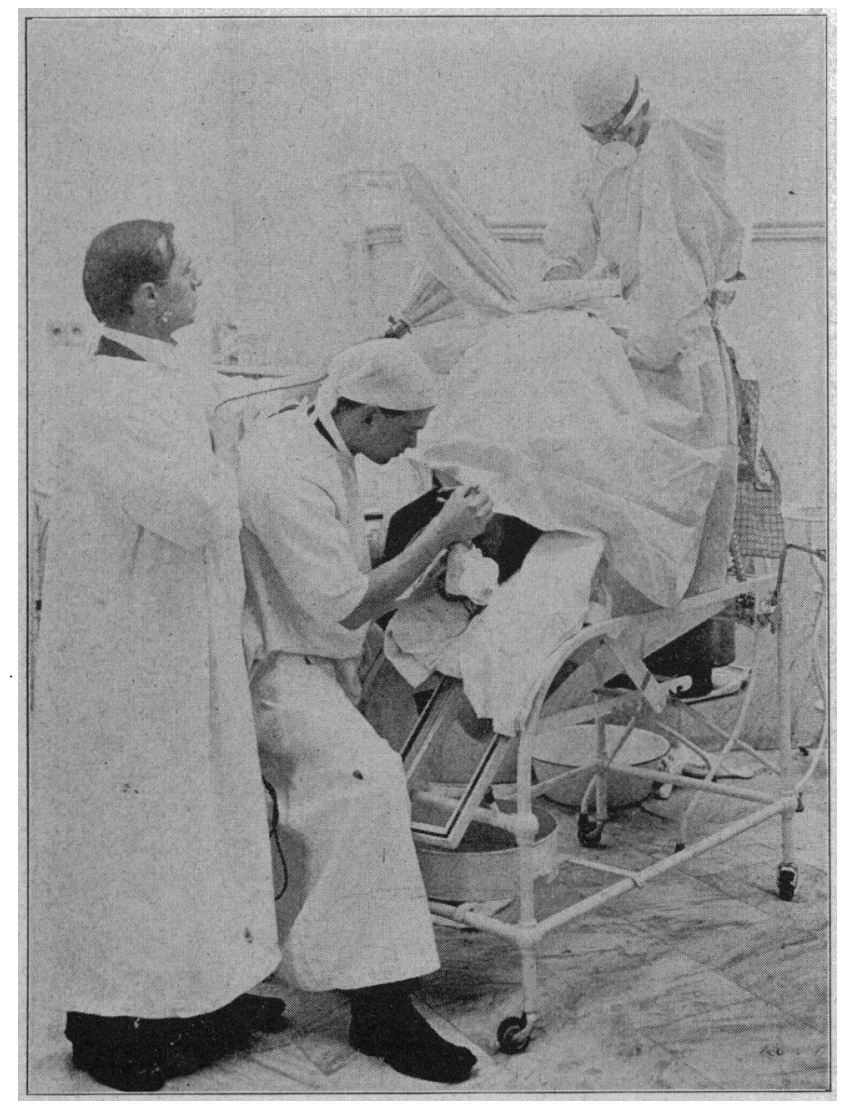

Fig. 2.-Method of examining the female bladder on the Boldt

urethra is dilated to the necessary size and a speculum containing its obturator introduced into the urethra.. By means of a crank the top of the table is turned on a transverse axis so that the lower end is elevated and the upper end depressed. The patient is thus made to rest on an inclined plane, being held by the shoulder supports, her trunk being flat against the table and not bent in any way, so that her respiration is free and the anesthetic easily administered. The writer usually raises the table-top until its lower end is twenty-three inches above the normal level. The obturator is then removed from the speculum, allowing air to enter and dilate the bladder. The examination of the bladder and ureters is then carried out, the examiner standing on a stool so that the eyes may be well above the outer end of the speculum.
This posture has all the advantages of the genupectora! position and none of its disadvantages. In difficult cases in which distension of the bladder has not been thoroughly satisfactory I have not been able to get better results by trying the genupectoral position. This method is also advantageous in the cases in which examination of the bladder or catheterization of the ureters is to be followed by operation. The table is merely lowered and the procedure at once begun if the vaginal route is to be chosen.

In the examination of the rectum also, the position is highly satisfactory, air distension being obtained in the great majority of cases as soon as the anus is opened. The various forms of rectal specula may be used with great ease. I have also found it serviceable for certain obstetric maneuvers for which the genupectoral position has hitherto been employed.

\section{THE DIFFERENTIAL DIAGNOSIS OF TYPHOID FEVER.*}

WILISON O. BRIDGES, M.D.

Professor of Medicine, University of Nebraska College of Medicine. OMAHA, NEB.

The combined group of symptoms which establishes the diagnosis of typhoid fever are: a gradually increasing fever with evening exacerbation and morning remission; general malaise with headache; a furred tongue with red edges and tip; nose bleed; a relatively slow pulse (possibly dicrotic) ; abdomina"l distension with tympany. gurgling and tenderness in the right iliac fossa on firm pressure; a roseolar eruption confined principally to the abdomen and chest; enlarged spleen, and the physical signs of bronchial catarrh.

Should every case of typhoid fever present the foregoing symptoms, the diagnosis would be simple and mistakes would not be common, but unfortunately this is not true and I believe the classical history, as portrayed in the books, is the exception rather than the rule. Because of the great variability in the symptoms and the tardiness of the appearance of many of them, the diagnosis is often not clear until the patient is far advanced in his illness. We have to wait a week from the commencement of the attack to demonstrate the character. istic fever chart, seven to ten days to find the rose spots, usually the same time to demonstrate an enlarged spleen; during this period what an anxious time the attending physician has in satisfying the family and friends that he is positive as to the nature of the trouble. Too often he starts off with la grippe, which does not respond in a few days to treatment. but turns out to be a malarial fever which later becomes complicated by typhoid at the first sign of an intestinal hemorrhage or a perforation.

Probably typhoid fever can be made to answer for a greater number of affections than any other disease. The only consolation the physician can find in his unfortunate position frequently is, that he is not the only one to be similarly situated; in fact, he is not even an exception, for we have all been there and will be again. The eminent men who were in attendance upon the Czar of Russia a year ago had him down with la grippe for nearly a week before typhoid fever showed up.

In quite a number of cases, the preponderance of some symptom leads away from the suspicion of the disease. and accounts for many errors in diagnosis. I have seen the physical signs of bronchitis so intense in the early

* Read before the Rotna Valley Medical Society, at Aroca, Iowa Feb. 13. 1902 . 
history of a case with accompanying high fever as to entirely mislead; the pain in the head so great as to make the attending physician certain of a case of meningitis, and obstinate constipation with distension and tenderness in the right iliac region are suggestive of appendicitis; and $I$ have myself a number of times, from the rapid onset and severity of chilliness, headache, general aching, fever and cough with acute catarrh of the whole respiratory tract, felt certain of a diagnosis of la grippe, only to find out later that I had guessed too soon. There seems no similarity whatever between a so-called walking case of typhoid fever and a case presenting most all the characteristic symptoms in their greatest severity, and within these two extremes what can not be represented? The physician who does not make snap diagnoses, or, properly speaking, "guesses"; who examines every case of disease carefully at each visit; who elicits a clear, concise history and always has typhoid fever in his mind when he finds a continued fever, will come out in the long run with fewer mistaries charged against him. It is not that we are not conversant with the diagnostic symptoms and signs of typhoid fever, but it is that we do not sufficiently apply that knowledge to the individual case.

It is important that the diagnosis be made at the earliest possible time, and yet this is not always practicable because of the tardiness of many prominent diagnostic symptoms. Fortunately for him, the physician is not consulted until the patient has ailed for several days, and the history will be presented of a slowly-developing affection which will be in marked contrast with most other acute diseases. I have come to regard a relatively slow pulse, which is so often present throughout the entire course of the disease, as strongly suggestive of typhoid and if it be found dicrotic in character the presumption will be quite strong. This is also in marked contrast to most diseases confounded with typhoid fever. The pulse in the first week rarely exceeds 90 , is often 80 or less, even in the presence of a temperature of 103 or more. Women and children are frequent exceptions to this rule.

An early sign almost invariably present is the diazobenzol urinary reaction of Ehrlich, which is commonly found as early as the fourth day. A few years ago the writer read a paper before the Missouri Valley Medical Society on the "Early Diagnosis of Typhoid Fever," in which his experience with this test, as applied to about 20 cases, was narrated, and his observations since, extending over about as many more, confirm his belief that the application of this test is not sufficiently frequent in practice, and that its value as a diagnostic sign is underestimated. The very fact that it may often be found by the fourth day, three days before the earliest rose spot, or palpable spleen, adds to its importance. True, it is found in malaria, in measles, in the latter stages of tuberculosis and in smallpox; but aside from malaria, these affections could hardly be confounded with typhoid fever. There is response to the test unti] about the eighteenth day of the fever. On the occurrence of a true relapse it reappears. I always keep the solutions on hand and have found it advantageous to carry them with me to suspected cases in the country. The test solutions are: 1. Sulphanilic acid, grains 20 ; hydrochloric acid, drams 3 ; distilled water, ounces 8; mix. 2. Sodium nitrite, grains 24; distilled water, ounce 1. 3. Aqua ammonia. 2 drams of No. 1, are mixed with 3 drops of No. 2 in a test tube; an equal quantity of the urine added and thoroughly shaken.
Eight drops of ammonia are then allowed to fall upon the foam; when a distinctly pink color of the latter occurs, to be followed by a bright carmine-red zone underneath. If the liquid be then poured into a white porcelain dish it will appear as a light yellowish red.

The Widal blood reaction is of considerable diagnostic importance, and when obtained in dilutions of forty or more, the certainty of the existence of typhoid fever in a suspected case may be entertained. Work along this line is demonstrating, however, that the reaction occurs in those who have previously had the disease, and this fact should be elicited before the deduction is made. It requires fresh cultures of the bacillus typhosus to make the test satisfactorily, and hence it is not always practicable to the country practitioner.

In cases where the test is essential to clear up an uncertainty, a coverslip of blood or serum can be readily sent to the bacteriological laboratory connected with any of our medical colleges. The typical reaction does not usually occur before the sixth or seventh day; exceptionally it is considerably later.

The rose spots vary within wide limits as to numbers and location. Oftentimes they are so few as to escape detection unless carefully searched for. Their diagnostic significance is based upon their disappearance under pressure, to reappear so soon as the pressure is vithdrawn; their non-elevation above the surface, and the individual spot having a life history of three days. They are occasionally found upon the arms and about the wrist. I believe from my own observation that they may be found in every case. Not appearing before the seventh day and occasionally as late as the tenth, they are of course not an early diagnostic sign.

The combined symptoms which are almost invariably present during the first week to be relied upon for a presumptive diagnosis are: a daily increasing temperature with headache and malaise; moist furred tongue with red edges and tip; a pulse of 80 or thereabouts and evidences of bronchial catarrh. If to these be added the diazo-benzol urinary reaction on the fourth to the sixth day with or without the occurrence of nose bleed, the presumption is very strong. A palpable spleen, rose spots and the Widal reaction early in the second week, supplementary to the foregoing, would make the diagnosis absolute.

The diseases most commonly mistaken for typhoid fever are: remittent malarial fever, continued fever of uncertain septic origin, commonly called simple febricula, acute miliary tuberculosis, la grippe, appendicitis, typhoid pneumonia, and septicemia.

Remittent fever or estivo-autumnal fever is the only form of continued fever of malarial origin. It is rarely seen outside of malarial districts and in this section of the country is scarcely known excepting in the arena of a mistaken diagnosis; and yet $I$ believe more cases of typhoid are treated for malarial fever than are treated for typhoid. This is a broad statement, but it is based upon observation and experience. The word malaria covers a multitude of sins, but it is inexcusable to carry it into a domain where recent knowledge and methods can so readily disarm. There are many points in common between these two fevers, but there are more points of difference. Malarial fever js usually ushered in by a distinct chill, often repeated, followed soon br hich fever with two daily exacerbations and remissions; typhoid by chilliness followed by gradual rise in fever with only one exacerbation and remission daily. The rapid pulse of commencing malaria is in marked con- 
trast with the slow pulse of typhoid. In the former there is rarely nose bleed, in the latter this is quite frequent. There are rarely abdominal symptoms in malaria, and there is commonly nausea and vomiting. The roseola is not found in malaria. The presence of the Widal blood reaction would be favorable to typhoid, and the crescentic plasmodium in the blood demonstrable by the microscope would be positive of malaria. An intestinal hemorrhage would be presumptive of typhoid and a marked susceptibility of the fever to quinin would savor of malaria. Simple febricula has not the nose bleed. slow pulse, diazo urinary reaction, palpable spleen, roseola and abdominal symptoms of typhoid, runs a shorter course and is much more readily influenced by treatment.

Acute miliary tuberculosis has not infrequently been confounded with typhoid fever. The onset of the former is more rapid and pronounced, the pulse rate much higher, greater frequency of respiration; there is not apt to be nose bleed, palpable spleen, rose spots; there is decided tendency to fine moist râles throughout the chest with loose cough not common to typhoid, and frequently the sputum will contain abundance of tubercle bacilli.

La grippe and typhoid fever prevail at the same season of the year. Mild cases of the latter are not unlike moderately severe cases of the former which have not early complications. La grippe is more sudden in onset, is usually consecutive to or coincident with a cold, has a more rapid pulse, the ferer is more susceptible to treatment responding in two or thrce days where sequelæ do not supervene; the diazo-reaction is absent, and there are no rose spots or palpable spleen.

Cases of prolonged appendicitis with slow onset will present differences only which require close investigation. The pulse here will be higher in proportion than the fever, the morning and evening temperature will vary less; there is no nose bleed, or roseola, or enlarged spleen, or bronchial catarrh, or diazo-reaction. There is more apt to be abdominal pains, muscular rigidity in right iliac region and moderate leucocytosis, which is much increased where pus is present.

Walking cases of typhoid with perforation of the intestine, are quite suggestive of fulminating appendicitis. and I have known errors of diagnosis to occur where, at operation, a perforated intestine and ulcerated Peyer's patches demonstrated the typhoidal character of the case. Intestinal perforation occurs at such a time in typhoid fever that a careful inquiry into the above differences in the symptoms and signs should leave no room for doubt, and although operation might be advisable in both, it is much the more satisfactory that the diagnosis be made beforehand. Appendicitis may complicate typhoid fever and develop when the abdominal symptoms are most pronounced. A case of this kind was reported to the American Medical Association at the meeting in Atlantic City by Dr. Siegfried Weiss of Vienna, reported in The Journal of Nov. 24,1900 . The case was that of a child $93 / 4$ years old, which was supposed first to have influenza, then typhoid : then on the development of signs of an inflammatory tumor in the appendical region, and leucocytosis, the former diagnoses were thrown over and a suppurative appendicitis accepted. Why the author discarded typhoid I can not understand, for the symptomatology included fever, bronchial catarrh, diazo-urinary reaction, enlarged spleen, rose spots, diarrhea and the Widal blood reaction with the development of the local signs of appendicitis only on the twelfth day of the illness. Was it not more likely a typhoid infection with involvement of the appendix? Why this complication is not more common puzzles us, for certainly the bacillus typhosus should have as ready access to the appendix as do foreign bodies, which enter from the intestine to excite inflammation. Perhaps it is more frequent than we think for, but undetected.

Typhoid pneumonia is a misnomer, for it suggests the presence of a typhoid infection in a type of pneumonia which is so severe as to present certain symptoms suggestive of the typhoid state, when in reality the latter does not exist. The disease starts in violently with severe chill, high fever, rapid pulse, pain in chest. frequent breathing, to be soon followed by low delirium, abdominal distension, involuntary evacuations, coma and death within a week or so. The frequent absence of cough and expectoration mislead. A careful examination of the chest will detect a solidified lung, and a marked leucocytosis will indicate no typhoid fever.

Septicemia of gradual onset presents a similarity of symptoms which lead to error, and especially when typhoid occurs in the puerperal state. In septicemia there is usually discoverable some focus of infection; it is attended by a higher relative pulse-rate; there is not the fever chart of typhoid. Frequently sweating occurs, the rose spots are absent as is also the Widal blood reaction.

The irregularity of typhoid fever in children. I believe, accounts for its supposed relative infrequency at this period of life. In many cases the onset is more abrupt; there is less apt to be complaint of general malaise; nose bleed is comparatively rare; nervous symptoms are more pronounced. 'The' abdominal symptoms, when present, are similar to those of enteric or gastro-enteric catarrh and especially in young children or infants are many of the symptoms wanting.

If the diazo-urinary test, and the Widal blood reaction and splenic enlargement and a search about the abdomen and chest for rose spots, were more carefully inquired into, in the continued fever of early life, we would find more typhoid. The loose movements of enteric catarrh are not like those of tuphoid. They are apt to be green; they contain mucus; sometimes blood and generally undigested food. Colicky pains frequently precede or attend the movements, all of which are not found in typhoid fever.

\section{GRAVE ABDOMINAT, INJURIES WITHOUT EXTERNAL EVIDENCES OF TRAU. MATISM.* \\ R. HARVLY REED, M.D.}

l'rofessor Fmeritus of Principles and Practice of Surgery and Clinical Surgery, Ohio Medical University; Division Surgeon, U.I.R.R Co.: Surgeon U. P. Coal Co.; Surgeon-General of Wyoming. ROCK SPRIXGS, WYO.

It has been my privilege during the last twenty years to have met with a class of grave abdominal injuries which has given little or no external evidences of traumatism. The patient in each instance has given a history of a severe injury which resulted in death in from 24 hours to 26 days after the traumatism. I will only report three of these cases in detail, as the others were practically duplicates and to make a full report would only take up valuable time without special advantage.

The first of these cases which came to my notice oc-

* Read before the Western Surgical and Gynecological Associa tion, at Chicago, Dec. 18-19, 1901. 